Bull. Mater. Sci., Vol. 2, Number 1, January 1980, pp. 71-78. (C) Printed in India.

\title{
Surface structures on second order prism faces of s ynthetic quartz crystals
}

\author{
M S JOSHI, K B SARAF and A V ANTONY \\ Physics Department, Sardar Patel University, Vallabh Vidyanagar 388120
}

MS received 8 October 1979; revised 17 December 1979

\begin{abstract}
Surface structure studies on various $\{1120\}$ (second order prism) faces of synthetic quartz crystals are reported. Horizontal striations and polygonal growth pyramids are predominant on these faces. Growth layers modified by the presence of guest microcrystals attached to the host second order prism faces are illustrated and explained. It is shown that microcrystals and impurities influence growth and development of second order prism faces of synthetic quartz crystals. Mechanism of growth of these faces is assessed in the light of the observed surface structures.
\end{abstract}

Keywords. Surface structures; second order prism faces ; synthetic quartz crystals.

\section{Introduction}

The study of surface structures of crystal faces offers a tool for understanding the growth and development of habit faces in particular and of the crystals in general. Sunagawa (1975) had shown that surface micrographs of crystal faces are far more sensitive to differences in growth conditions than macromorphology. Joshi and Vagh (1966) reported the role of spirals in growth of prism faces of cultured quartz. Morphological descriptions and surface growth features of synthetic quartz were also given among others by Bandyopadhyay and Saha $(1966,1968)$, Joshi and Vagh (1964) Joshi and Kotru (1968).

In natural quartz crystals, $\{10 \overline{1} 0\}$ (first order prism) faces usually show horizontal striations perpendicular to $c$-axis and $\{10 \overline{1} 1\}$ (rhombohedral) faces show triangular growth pyramids. However, the situation is different in cultured quartz crystals. Surface structures do vary, depending upon crystallising conditions, existence dislocations, impurities, stacking faults, etc. Therefore surface topographical studies of $\{11 \overline{2} 0\}$ (second onder prism) fases of synthetic quartz crystals were undertaken to understand the growth mechanism of second order prism faces of synthetic quartz crystals. The present paper concerns only with the study of surface structures on second order prism faces of synthetic quartz crystals.

\section{Experimental}

The crystals secured from USA were grown on different cut seeds. Crystals were thoroughly cleaned with the usual reagents, silvered in a vacuum coating unit and 
then examined under a metallurgical microscope. The technique of light profle microscopy developed by Tolansky (1952) was employed to verify the observed features.

\section{Observations and discussion}

Figure 1 illustrates the usual horizontal striations on a second order prism face of synthetic quartz crystal. These striations are perpendicular to $c$-axis of the crystal. In several cases it was observed that the prism faces exhibit polygonal growth hillocks on them. One such case is shown in figure 2. The summit of the pyramid acts as a growth centre, from which growth layers initiate and pile up. Growth layers composing such hillocks are visible in figures $3 \mathrm{a}$ and $3 \mathrm{~b}$. No spiral growth hillocks were observed. This means that independent growth takes place on such faces by two-dimensional nucleation process and not by screw dislocation mechanism. Impurities or clusters of nuclei should have played a role in the formation of such hillocks. In many cases, prism faces exhibit a large number of growth centres from which growth layers originate. Bunching of growth layers composing a hillock is also observed. One such case is shown in figure 4. Such growth fronts are almost a common feature on such faces.

Growth is also influenced by the presence of microcrystals attached to the growing second order prism faces of quartz crystals. Figure 5 illustrates such a case. During growth of the crystals clusters of nuclei or presence of impurities will lead to formation of microcrystals. Some of these get attached to the growing crystal face. Several faces were observed in which microcrystals attached to the prism face initiate growth layers. A typical case, where growth layers are initiated from the tips of microcrystals is shown in flgure 6. Most of the microcrystals observed on the host prism faces were found to be misoriented with respect to the host face. Sometimes a microcrystal which gets attached to the growing face may also retard the growth of the face. Figure 7 shows that the microcrystal attached on to the face has retarded the growth on the prism face on the other side of the microcrystal.

If a guest crystal is attached to a host face with its $c$-axis either perpendicular or inclined to the host crystal face and remain attached for a longer time, it should provide a big kink for further growth to take place around it. If a guest crystal gets detached from the host face, hexagonal cavities appear on the face. One typical case is shown in figure 8 . When the attached microcrystal is inclined to the host face, growth layers are initiated from the portion of the crystal touching the face. Further growth on the crystal face slowly incorporates the microcrystal into the body of the crystal as shown in figure 9. In several cases it was observed that a number of growth centres are initiated at several points on a microcrystal attached parallel to the face. As growth proceeds for some time around the microcrystal, growth fronts of growth layers composing growth pyramids will spread over the attached microcrystal also. When they cross over the microcrystal they get modified showing step-like structures as depicted in figure 10. Further growth of the face will lead to incorporation of microcrystals into the host crystal.

Our studies clearly indicate that second order prism faces of synthetic quartz crystals develop predominantly by two-dimensional spreading of growth layers. 

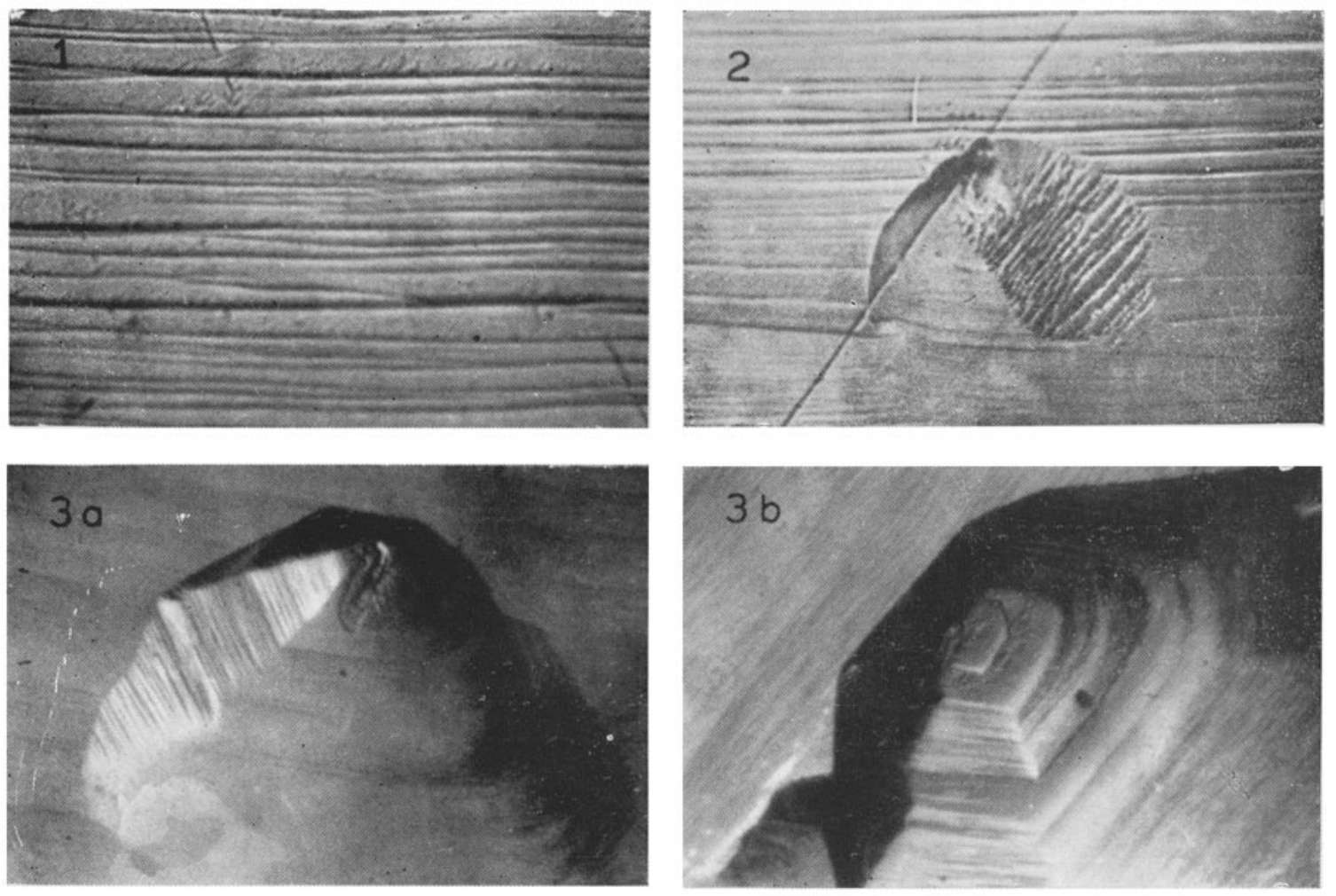

Figures 1-3. 1. Horizontal striations $(\times 136)$. 2. A typical growth pyramid $(X 150)$. 3a, and 3b. Spreading of growth layers around a growth pyramid $(\therefore 85$ and $\times 165)$. 

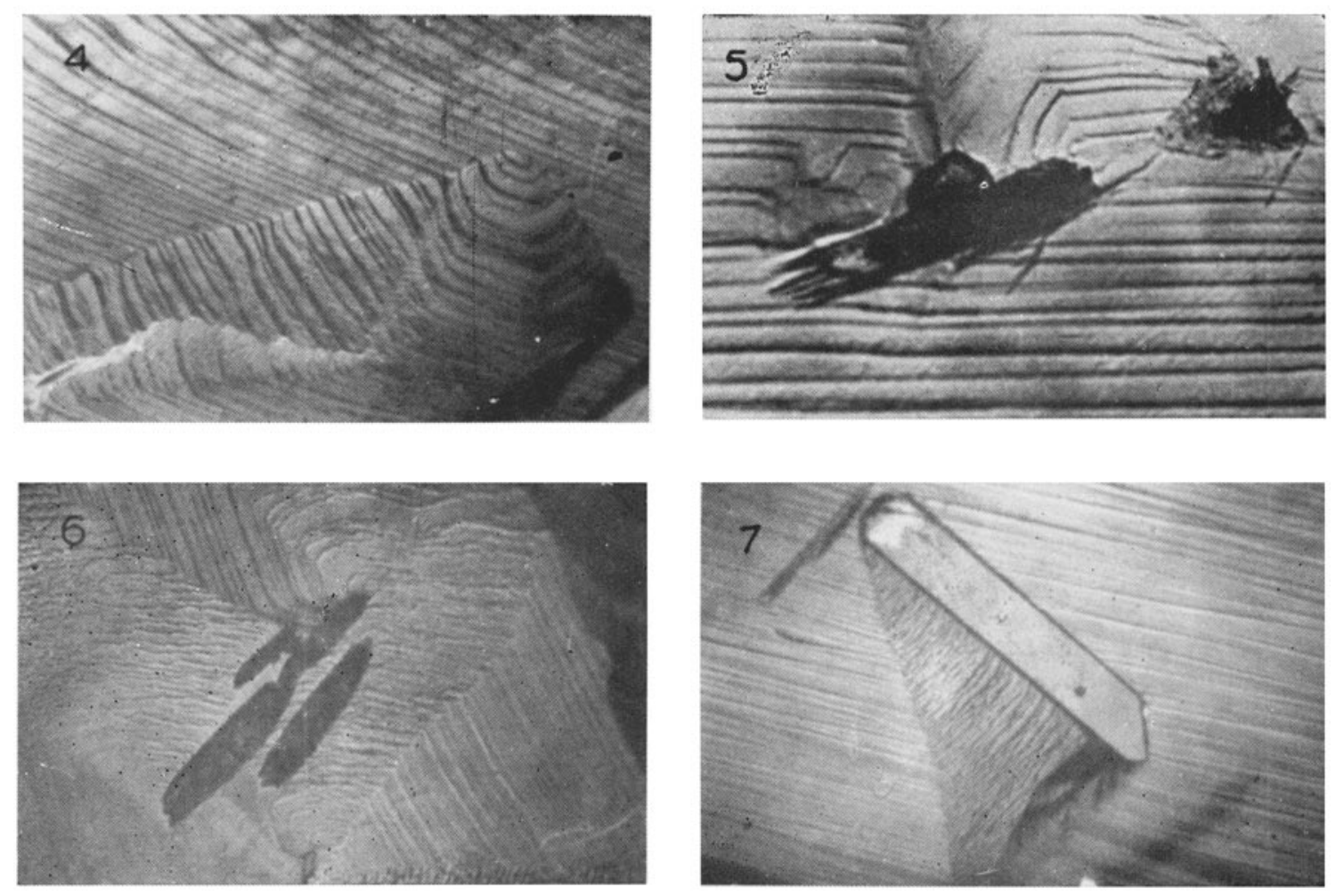

Figures 4-7. 4. Bunching of grow th layers $(x, 110)$. 5. Growth layers modified due to an attached microcrystal $(x$ 415). 6. Growth layers initiated from tips of microcrystals $(\times 120)$. 7. Growth of a face-retarded by an attached microcrystal ( $\times 190)$. 

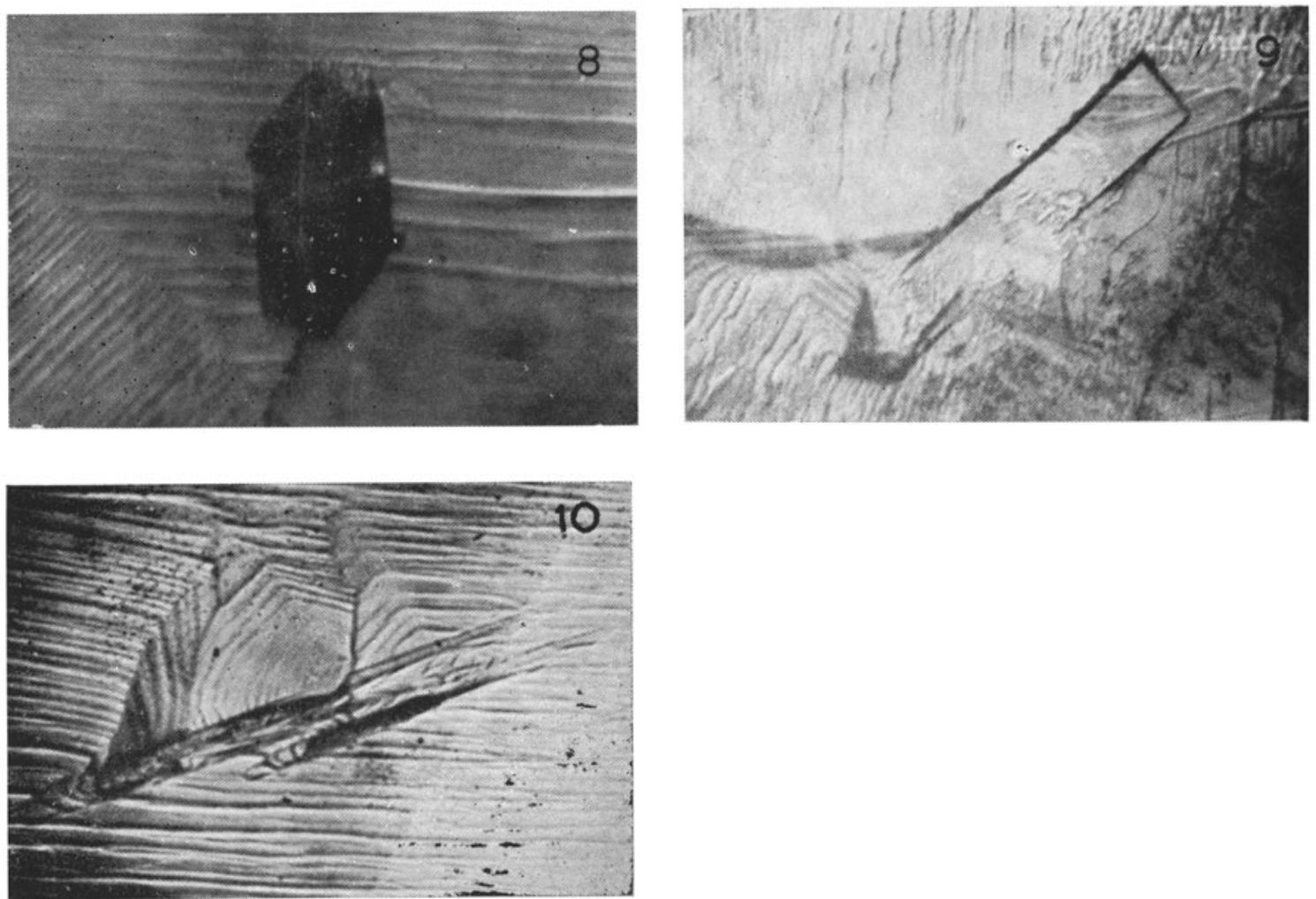

Figures 8-10. 8. Hexagonal cavity due to detachment of a microcrystal $(\times 235)$. 9. Growth around a microcrystal inclined to the host face $(\times 235)$. 10. Spreading of growth layers leading to incorporation of microcrystals into the host crystal $(x 210)$ 
Second order prism face is not a habit face of a quartz crystal. However, it is found occasionally on synthetic quartz crystals. Its origin could be explained as follows :

(i) It may develop as a result of growth by spreading of growth layers on its neighbouring habit faces. (ii) Independent growth on it may take place at impurities which can play the role of growth nuclei. This is possible at any stage of growth process of the crystal. Islands of molecules absorbed on such a face can also lead to further growth and development of a second order prism face. In these two cases growth pyramids should be expected to exist on the face. (iii) Guest microcrystals on a second order prism face can also play the role of growth nuclei. This is likely to happen more towards the end of growth of the face. In the present investigation evidence has been obtained to support both the possibilities (ii) and (iii) given above.

Changes in surface structures due to presence of microcrystals suggest that microcrystals nucleated during the growth of crystals will influence growth and development of second order prism faces of quartz crystals.

It is thus concluded that second order prism faces of synthetic quartz crystals grow mainly by two-dimensional spreading and piling up of growth layers.

\section{Acknowledgements}

The authors are thankful to Dr Baldwin Sawyer (Sawyer Research Products, USA) for the supply of quartz crystals and to Dr P N Kotru, University of Jammu, for discussions and suggestions. One of us (KBS) is thankful to the University Grants Commission, New Delhi, for a fellowship and to the Principal, Pratap College, Amalner, for study leave. AVA is thankful to the Council of Scientific and Industrial Research, New Delhi, for a fellowship.

\section{References}

Bandyopadhyay $T$ and Saha P 1966 Cent. Glass Ceram. Res. Inst. Bull. 1359

Bandyopadhyay $\mathrm{T}$ and Saha P 1968 Indian J. Phys. 42595

Joshi M S and Kotru P N 1968 Am. Miner. 53825

Joshi M S and Vagh A S 1964a Indian J. Pure Appl. Phys. 2346

Joshi M S and Vagh A S 1964b Am. Miner. 491771

Joshi M S and Vagh A S $1966 J$. Appl. Phys. 37315

Sunagawa I 1975 Fortschr. Mineral 52515

Tolansky S 1952 Z. Electrochem. 56263 